EPIGENETICS

\section{Breaking the silence}

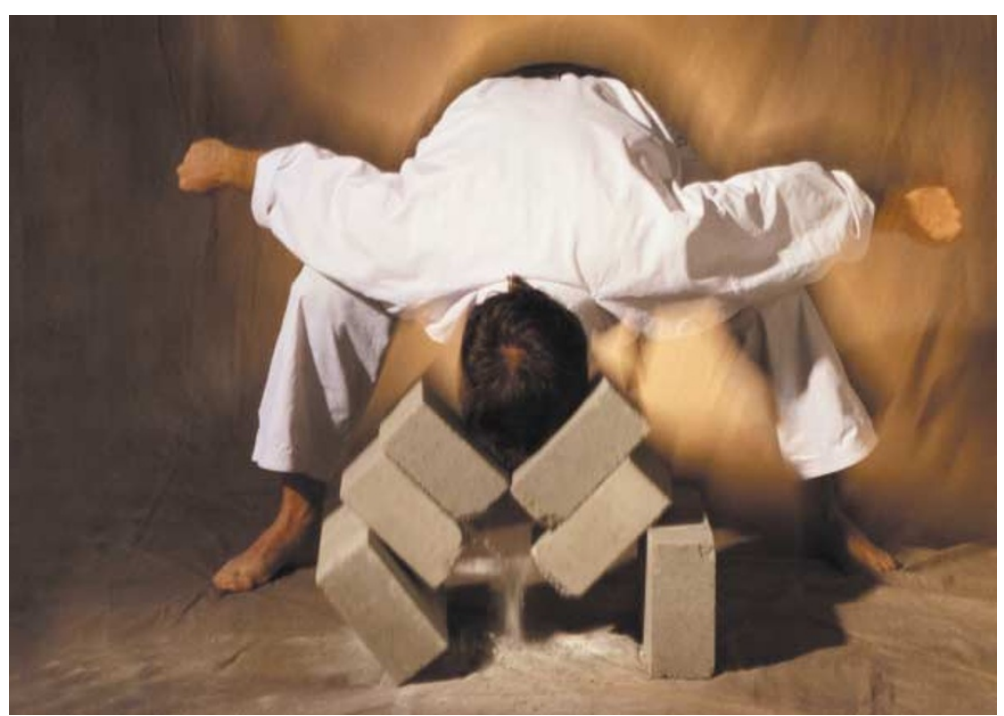

As well as deleting or mutating tumour-suppressor genes, cancer cells can turn off their expression by epigenetic means. It has been a challenge, however, to determine exactly which genes are transcriptionally silenced in a particular tumour. A new microarray-based screen can identify genes that are suppressed by hypermethylation and histone deacetylase (HDAC) activity, revealing 74 genes that are downregulated by this process in colorectal cancer cells.

Epigenetic gene silencing involves increased methylation of DNA sequences known as CpG islands, which usually lie in gene promoter regions, and also the activity of HDACs, which control chromosome condensation. But because the sites that are regulated by these mechanisms are often outside the coding regions of genes, it is laborious to search for nearby genes and determine whether their expression patterns are altered. Sometimes, it is a challenge to even identify hypermethylated sites, as some of these do not contain $\mathrm{CpG}$ islands, and there is no established method to identify genes that are regulated by HDAC activity.
Hiromu Suzuki et al. have overcome these obstacles by using microarrays to compare the expression patterns of untreated cells with those treated with 5-aza-2' deoxycytidine (DAC), which blocks DNA methylation, and/or trichostatin A (TSA), which inhibits HDAC activity. A screen of over 10,000 genes in eight different colorectal cancer cell lines revealed 74 genes that were upregulated by treatment with DAC and/or TSA. Of these genes, 51 required treatment with both drugs for suppression to be relieved, whereas 23 upregulated expression after treatment with TSA alone. So most genes seem to require a combination of hypermethylation and HDAC activity to be silenced.

Many of these genes were already known to be involved in tumorigenesis, such as SEZ6L, a gene of unknown function that is mutated in lung and breast cancer, and TIMP2, an inhibitor of the matrix metalloproteinase family of genes that are required for cell invasion. One unexpected finding

\title{
Controlled destruction
}

There have been many attempts to direct the cytolytic activity of adenoviruses towards tumours. The tricky part, however, is manipulating them to kill cancer cells but not normal cells. A recently engineered adenovirus, ONYX411 , only lyses cells that lack a functional form of the tumour suppressor retinoblastoma $(\mathrm{RB})$ - making it a safer and more effective oncolytic virus.

Oncolytic adenoviruses caused much excitement when they were first described in 1996. ONYX-015 - the first oncolytic virus shown to preferentially kill cells in which $\mathrm{p} 53$ is inactivated - reduced tumour growth in mice and is, at present, in clinical trials for the treatment of head and neck cancer. One drawback of this agent, however, is that it must be directly injected into the patient's tumour, because it has been shown to have toxic effects when administered intravenously. This limits its use to patients who have large accessible tumours.
Leisa Johnson et al. have improved on the oncolytic virus concept to create a version that can be applied systemically. The new virus, ONYX-411, was engineered to propagate only in cells with defects in the RB pathway - which includes almost all cancer cells. So, how does ONYX-411 work? The authors placed the adenoviral $E 1 A$ and $E 4$ genes - which are required for viral propagation - under control of the human cellular E2F1 promoter. This promoter is activated by $\mathrm{E} 2 \mathrm{~F}$ and is selectively active in cancer cells that contain high levels of E2F owing to loss of RB activity. The E1A gene of ONYX-411 also contains a point mutation that prevents its interaction with RB, although it can still mediate S-phase entry. This mutant form of E1A therefore cannot disrupt RB activity in normal cells, and only replicates in cells that lack functional RB.

The authors tested ONYX-411 and found that it killed many different types of cancer cells, but not normal cells, in vitro. But can it be safely administered intravenously? A detailed analysis in mice revealed that ONYX-411 caused less systemic toxicity than all other adenoviral vectors it was compared with, including ONYX-015. But most importantly, intravenously administered ONYX-411 prolonged survival of mice bearing human cervical cancer xenografts, curing $60-70 \%$ of mice without damage to normal tissues.

Although the mechanisms responsible for the lower systemic toxicity associated with ONYX-411 are not clearly understood, the multiple systems for ensuring that the virus replicates only in RB-deficient cells are likely to be responsible. ONYX-411 might therefore be useful for treating metastatic tumours and other cancers that require systemic administration of therapy.

\section{Kristine Novak}

\section{(4) References and links}

ORIGINAL RESEARCH PAPER Johnson, L. et al. Selectively replicating adenoviruses targeting deregulated E2F activity are potent, systemic antitumor agents. Cancer Cell 1, 325-337 (2002)

FURTHER READING Dubensky, T. W. Engineering tumor cellselective replicating adenoviruses: a step in the right direction toward systemic therapy for metastatic disease? Cancer Cell toward systemic therapy for metastatic disease? Cancer Cel fringe or cutting edge? Nature Rev. Cancer 1, 130-141 (2001) WEB SITE

Frank McCormick's lab:

http://cc.ucsf.edu/people/mccormick_frank.html 